\title{
Determinación del consumo de agua del duraznero por lisimetría
}

\author{
Lucía Puppo ${ }^{1} \&$ Mario García-Petillon
}

\begin{abstract}
RESUMEN
En el año 2004 se instaló un lisímetro de compensación de 2 × 2 × 0,80 m con capa freática constante en un monte de durazneros, con el objetivo de estudiar el consumo de agua de ese cultivo en la región sur del Uruguay. Las mediciones se hicieron en las tres primeras temporadas de crecimiento. El consumo en la primera temporada alcanzó valores de $5 \mathrm{~mm} \mathrm{~d}^{-1}$, equivalente a $56 \mathrm{~L}$ en el marco de plantación. En la segunda temporada llegó a $6 \mathrm{~mm} \mathrm{~d}^{-1}$ (68 L), aunque alcanzó valores extremos de más de $7 \mathrm{~mm} \mathrm{~d} \mathrm{~d}^{-1}$. Cuando el árbol alcanzó su tamaño adulto, el consumo máximo se mantuvo alrededor de los $6 \mathrm{~mm} \mathrm{~d}^{-1}$, aunque este valor se alcanzó más temprano que en la temporada anterior. Este adelanto se correspondió con un mayor índice de área foliar (IAF). El coeficiente de cultivo $\left(\mathrm{K}_{\mathrm{c}}\right)$ fue de alrededor de 1,2 en la primera temporada, y de 1,4 en las dos siguientes. Se calcularon los coeficientes de base $\left(K_{\mathrm{cb}}\right)$ y su valor se ajustó por el modelo tri-segmentado. Su valor fue de 0,91 en la primera temporada, 1,04 en la segunda y 1,20 en la tercera. Se hacen algunas observaciones metodológicas sobre el uso de este lisímetro, proponiéndose incorporar la variación de agua en el suelo a la ecuación de balance de volúmenes y se propone adicionar riego desde la superficie.
\end{abstract}

Palabras-clave: coeficiente basal de cultivo, coeficiente de cultivo, evapotranspiración, Prunus persica L. Batsch, riego

\section{Determination of water use in peach trees with a lysimeter}

\begin{abstract}
A compensation lysimeter with constant freatic water table of $2 \times 2 \times 0.80 \mathrm{~m}$ was installed in 2004 in a peach grove, in order to study the crop water consumption in the south of Uruguay. Measurements were taken in the first three growing seasons. Evapotranspiration at the first season reached $5 \mathrm{~mm} \mathrm{~d}^{-1}$, equivalent to $56 \mathrm{~L}$ in the whole area. In the second season, it went up to $6 \mathrm{~mm} \mathrm{~d}^{-1}(68 \mathrm{~L})$, reaching extreme values of $7 \mathrm{~mm} \mathrm{~d}^{-1}$ and more. When the tree reached its adult size, the maximum consumption remained around $6 \mathrm{~mm} \mathrm{~d}^{-1}$, although this value was reached earlier than the season before. This early behavior came together with higher leaf area index (LAI). The crop coefficient $\left(\mathrm{K}_{c}\right)$ was around 1.2 in the first season, and 1.4 in the following two seasons. Basal crop coefficient $\left(\mathrm{K}_{\mathrm{cb}}\right)$ was calculated and its value was adjusted by the tri-segmented model. The value was 0.91 at the first season, 1.04 in the second and 1.20 in the third. Some methodological observations are made regarding the lysimeter use. Adding the soil water changes to the mass balance equation is suggested, as well as incorporating irrigation from the surface.
\end{abstract}

Key words: basal crop coefficient, crop coefficient, evapotranspiration, Prunus persica L. Batsch 


\section{INTRODUCCIÓN}

El conocimiento de la evapotranspiración de los cultivos $\left(\mathrm{ET}_{\mathrm{C}}\right)$ es esencial para un manejo eficiente del riego ajustando el volumen y la frecuencia del riego a los requerimientos de los cultivos. Un manejo correcto del riego permite apuntar a rendimientos altos, con estabilidad entre años, y a una calidad óptima del producto cosechado, haciendo posible un uso racional del agua y de la energía, minimizando el desperdicio de ambos recursos y la contaminación del medio ambiente. Asimismo este dato es fundamental para un correcto diseño de los sistemas de riego.

Esto es de resaltar cuando se considera lo reportado por García-Petillo et al. (2003), en cuanto a que los productores frutícolas de la zona Sur del país no riegan en cantidad suficiente como para cubrir los requerimientos del cultivo y obtener rendimientos diferenciales en la cantidad y calidad de la producción que justifiquen el costo del riego. Este riego insuficiente es consecuencia de fuentes de menor capacidad a las requeridas y/ó equipos subdimensionados, así como del desconocimiento por parte de los productores en lo referente a un correcto manejo del riego.

A pesar de lo relevante del tema, en el Uruguay no existe investigación en el consumo de agua de los cultivos, siendo la excepción un trabajo en citrus para el Sur del país (García-Petillo \& Castel, 2007).

Existen diversos métodos tanto para medir como para estimar la $\mathrm{ET}_{\mathrm{c}}$. Los lisímetros son dispositivos que contienen un volumen de suelo, aislado hidrológicamente del suelo circundante, en el cual resulta posible controlar y medir los diferentes términos que intervienen en el balance. Dentro de estos, los lisímetros de pesada sirven para medir la ET durante intervalos cortos de tiempo (una hora ó menos). Su sensibilidad puede ser de 0.03 ó $0.05 \mathrm{~mm}$ de agua pero con un costo muy alto que lo hace inviable para el Uruguay.

Existen diversos métodos para predecir la $\mathrm{ET}_{\mathrm{C}}$ dada la dificultad de obtener mediciones directas y exactas en condiciones reales. Algunos de ellos son válidos, únicamente, para las condiciones climáticas y agronómicas para las cuales fueron desarrollados.

La FAO (Allen et al., 1998), propone un doble paso de estimación, calculando primero la evapotranspiración del cultivo de referencia $\left(\mathrm{ET}_{\mathrm{o}}\right)$ y considerando luego la especificidad del cultivo a través de un coeficiente de cultivo $\left(\mathrm{K}_{\mathrm{c}}\right)$.

Los coeficientes de cultivo se pueden ajustar como $\mathrm{K}_{\mathrm{c}}$ medios ó como $\mathrm{K}_{\mathrm{c}}$ duales, siendo (Wright, 1982; Allen et al., 2005b):

$$
\mathrm{K}_{\mathrm{c}}=\mathrm{K}_{\mathrm{cb}}+\mathrm{K}_{\mathrm{e}}
$$

donde:

$\mathrm{K}_{\mathrm{cb}}$ - coeficiente base del cultivo

$\mathrm{K}_{\mathrm{e}}$ - coeficiente de evaporación del agua del suelo

$\mathrm{El} \mathrm{K}_{\mathrm{cb}}$ es definido como la relación entre $\mathrm{ET}_{\mathrm{c}} / \mathrm{ET}_{\mathrm{o}}$ cuando la superficie del suelo está seca, no existiendo evaporación, pero la transpiración se da a la tasa potencial dado que el agua no es limitante para el proceso.

El $\mathrm{K}_{\mathrm{e}}$ describe el componente de evaporación de la $\mathrm{ET}_{\mathrm{C}}$ que se da en la fracción húmeda y expuesta del suelo, a 0,1-0,2 m de profundidad. Los valores menores de profundidad corresponden a suelos arenosos y los valores mayores a suelos de francos a pesados.

Pereira (2004) y Allen et al. (2005a) presentan criterios para seleccionar el método de cálculo de los coeficientes de cultivo. Proponen que se utilicen los coeficientes culturales de base $\left(\mathrm{K}_{\mathrm{cb}}+\mathrm{K}_{\mathrm{e}}\right)$ en lugar de los coeficientes culturales medios $\left(\mathrm{K}_{\mathrm{c}}\right)$ cuando se trata de trabajos con fines de investigación, así como en la conducción del riego en tiempo real con sistemas de alta frecuencia, sobre todo en regiones con precipitaciones frecuentes.

El valor del $\mathrm{K}_{\mathrm{e}}$ es máximo luego de un riego o lluvia y decrece a medida que aumenta la cantidad de agua evaporada acumulada en la profundidad de suelo que interviene en la evaporación. El valor de este coeficiente nunca puede ex-

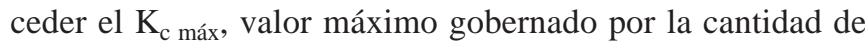
energía disponible en la superficie del suelo, limitado a su vez por la fracción de suelo húmeda y expuesta (few).

Los objetivos de este trabajo fueron:

1) Medir el consumo de agua de una planta de duraznero durante varios ciclos de crecimiento, desde su implantación hasta su tamaño definitivo.

2) Determinar el coeficiente de cultivo $\left(K_{c}\right)$ y el coeficiente de base $\left(\mathrm{K}_{\mathrm{cb}}\right)$ para la zona frutícola Sur del Uruguay.

\section{MATERIAL Y MÉTODOS}

A comienzos del 2004 se instaló en el Centro Regional Sur de la Facultad de Agronomía (34 40’ S, 56º $00 \mathrm{~W}$ ), en Canelones, Uruguay, un lisímetro de compensación con capa freática constante, inmediatamente después de la implantación de un monte de duraznero (Prunus persica L. Batsch) cv. Dixiland, con el que se midió el consumo de agua durante las temporadas 2004-2005, 2005-2006 y 2006-2007. El monte se plantó a 4,5 m entre filas y 2,5 m entre plantas, determinando un marco de plantación de $11,25 \mathrm{~m}^{2}$ y una densidad de 889 árboles ha-1 .

El lisímetro, instalado dentro del monte, consta de un macetón de PVC de $1 \mathrm{~mm}$ de espesor de pared, cuyas dimensiones son $2 \times 2 \times 0,8 \mathrm{~m}$ de profundidad, enterrado, asegurando que el mismo quede nivelado. El mismo es un lisímetro de capa freática constante, de estructura simple y bajo costo, desarrollado por el Departamento de Agronomía y Gestión del Agroecosistema de la Universidad Pisa, utilizado hasta el momento para cultivos hortícolas en invernáculos (Magnani et al., 1990; Bertolacci \& Megale, 1991), al cual se le hicieron las modificaciones necesarias, en la instalación, para adaptarlo a los intereses de este estudio.

En el fondo del macetón se mantiene una capa libre de agua de altura constante, que por capilaridad compensa el consumo de agua del árbol de duraznero confinado en la estructura. La altura de la capa se mantiene en un mismo nivel, gracias a un sistema de alimentación de agua que tiene lugar a través de un tubo instalado en el macetón y que contabiliza la cantidad de agua erogada, cerrándose una válvula cada vez que la altura de la capa alcanza el nivel prefijado de $12 \mathrm{~cm}$. 
Para asegurar el drenaje en el interior del macetón se realizó el rellenado del mismo manteniendo una secuencia granulométrica diferencial desde el fondo hasta la superficie como sigue: $5 \mathrm{~cm}$ de pedregullo, $2 \mathrm{~cm}$ de arena, $2 \mathrm{~cm}$ de una mezcla $75 \%$ arena y $25 \%$ de suelo franco, $2 \mathrm{~cm}$ de $50 \%$ arena y $50 \%$ suelo franco, $2 \mathrm{~cm}$ de $25 \%$ arena y $75 \%$ suelo franco y el resto hasta la superficie con suelo franco dispuesto en capas de a $2 \mathrm{~cm}$ compactadas suavemente con un pisón de madera.

Si bien los lisímetros con rellenos monolíticos representan mejor las condiciones naturales del campo y por lo tanto deben ser preferidos sobre todo en suelos estratificados, los lisímetros de relleno como el utilizado en este trabajo permiten obtener datos confiables de ETo y consumo de agua de los cultivos que no estén sometidos a condiciones de estrés (Aboukhaled et al., 1986).

Para que el sistema de alimentación funcione adecuadamente requiere de una cierta carga, para lo que se colocó un tanque elevado cuyo fondo se encuentra a $3 \mathrm{~m}$ por encima de la entrada de agua.

El lisímetro cuenta además con un sistema para evacuar los excesos de agua que puedan ocurrir con las lluvias, el cual consiste en un tubo abierto que permite la salida del agua por encima del nivel de la capa recolectándose en un tanque enterrado cuyo fondo está a $1 \mathrm{~m}$ por debajo del fondo del macetón. Este tanque se vaciaba después de cada medida.

En la temporada 2004-2005 se instalaron en el lisímetro dos tensiómetros a 15 y $30 \mathrm{~cm}$ de profundidad. En la temporada 2005-2006 se agregó un tercer tensiómetro a 60 cm.

Se determinó la curva de retención del suelo de relleno del lisímetro, utilizando la olla de presión.

Diariamente se registró:

- volumen de agua de entrada al lisímetro con un contador volumétrico

- precipitaciones ocurridas, con un pluviómetro de 0,20 m de diámetro ubicado a $30 \mathrm{~m}$ de la instalación

- volumen de drenaje con una escala calibrada

- volumen de entrada de agua al tanque elevado con un contador volumétrico

- tensión de agua en el suelo con tensiómetros

La $\mathrm{ET}_{\mathrm{C}}$ se calculó por balance de volúmenes mediante la fórmula:

$$
\mathrm{ET}_{\mathrm{c}}=\mathrm{R}+\mathrm{PP}-\mathrm{D} \pm \Delta \mathrm{Hs}
$$

siendo:

$$
\begin{aligned}
& \mathrm{ET}_{\mathrm{C}} \text { - evapotranspiración real del duraznero } \\
& \mathrm{R} \text { - riego } \\
& \mathrm{PP} \text { - precipitación sobre el lisímetro } \\
& \mathrm{D} \text { - drenaje del lisímetro } \\
& \Delta \mathrm{Hs} \text { - Variación de la humedad del suelo }
\end{aligned}
$$

La metodología utilizada (Magnani et al., 1990; Bertolacci \& Megale, 1991) considera que el volumen de suelo del lisímetro siempre está a capacidad de campo (CC) o muy próximo, al comienzo de cada ciclo diario, por lo tanto $\Delta \mathrm{Hs}=0$.

El volumen de la copa se calculó con una frecuencia mensual durante las tres estaciones de crecimiento evaluadas, a partir de medidas de altura, diámetro longitudinal y diámetro transversal.
Con la misma frecuencia se estimó el área total de hojas. Para ello se contó la totalidad de las hojas y se midió el área de una muestra de 100 hojas. Se calculó el Índice de Área Foliar (IAF) dividiendo el área total de hojas entre el marco de plantación, 11,25 m² en este caso. En los mismos momentos se midió intercepción de radiación con un ceptómetro PAR Sunfleck.

A fin de verificar que la planta del lisímetro estuviese en condiciones similares que las otras plantas del monte, se midió el potencial hídrico con cámara de presión, según lo reseñado por Boland et al. (1993) y siguiendo las precauciones de uso del método enumeradas por Hsiao (1990). Asimismo se midió conductancia estomática con un porómetro marca DELTA-T, modelo AP4.

Se calculó la $\mathrm{ET}_{\mathrm{o}}$ con la fórmula Penman-Monteith modificada por FAO (Allen et al., 1998), utilizando los datos climáticos diarios de la Estación Experimental Las Brujas (34 40' S; 56 $26^{\circ}$ ' W), ubicada a $15 \mathrm{~km}$ del sitio del ensayo.

Se ajustó el coeficiente de cultivo $\left(\mathrm{K}_{\mathrm{c}}\right)$, como el cociente entre la $\mathrm{ET}_{\mathrm{c}}$ medida en el lisímetro y la ETo-PM.

Se separó la evaporación directa del suelo $\left(\mathrm{E}_{\mathrm{s}}\right)$ realizando un balance hídrico de paso diario en los $15 \mathrm{~cm}$ superiores de la fracción del suelo húmeda y expuesta del lisímetro (few), siguiendo la metodología de Pereira (2004). Esta metodología demostró un buen desempeño al ser validada con datos observados de campo por Allen et al. (2005a) y Godinho et al. (2007). La fracción húmeda y expuesta se calculó como:

$$
\text { few }=1-\left(\frac{A s * \text { Rint }}{A l}\right)
$$

siendo:

As - área del lisímetro sombreada por la copa

Rint - fracción de la radiación interceptada por la copa al mediodía

$\mathrm{Al}$ - área del lisímetro

En forma complementaria, Goodwin et al. (2006) utilizaron la fracción de la radiación interceptada por la copa, en combinación con fotografías de la copa desde la dirección del sol, para estimar el área efectiva de sombreado, ajustando una relación entre ésta y la transpiración del árbol.

La transpiración directa de la planta se calculó como $\mathrm{T}=\mathrm{ET}_{\mathrm{C}}-\mathrm{E}_{\mathrm{S}}$ (Johnson et al., 2004). Por lo tanto, el coeficiente basal del cultivo $\left(\mathrm{K}_{\mathrm{cb}}\right)$ se calculó como $\mathrm{K}_{\mathrm{cb}}=\left(\mathrm{ET}_{\mathrm{c}}-\mathrm{E}_{\mathrm{s}}\right) / \mathrm{ET}_{\mathrm{o}}$.

Se ajustó la curva del Kcb mediante el modelo tri-segmentado, lineal- plateau-lineal (Allen et al., 1998; Ayars et al., 2003).

\section{RESULTADOS Y DISCUSIÓN}

\section{Consumo}

El consumo en la primera temporada (2004-2005), en que la planta tenía sólo un año de instalada, alcanzó valores de $5 \mathrm{~mm} \mathrm{~d}^{-1}$, equivalente a $56 \mathrm{~L}$ para el marco de plantación de 11,25 $\mathrm{m}^{2}$ (Figura 1).

En la segunda temporada (2005-2006), el consumo llegó 

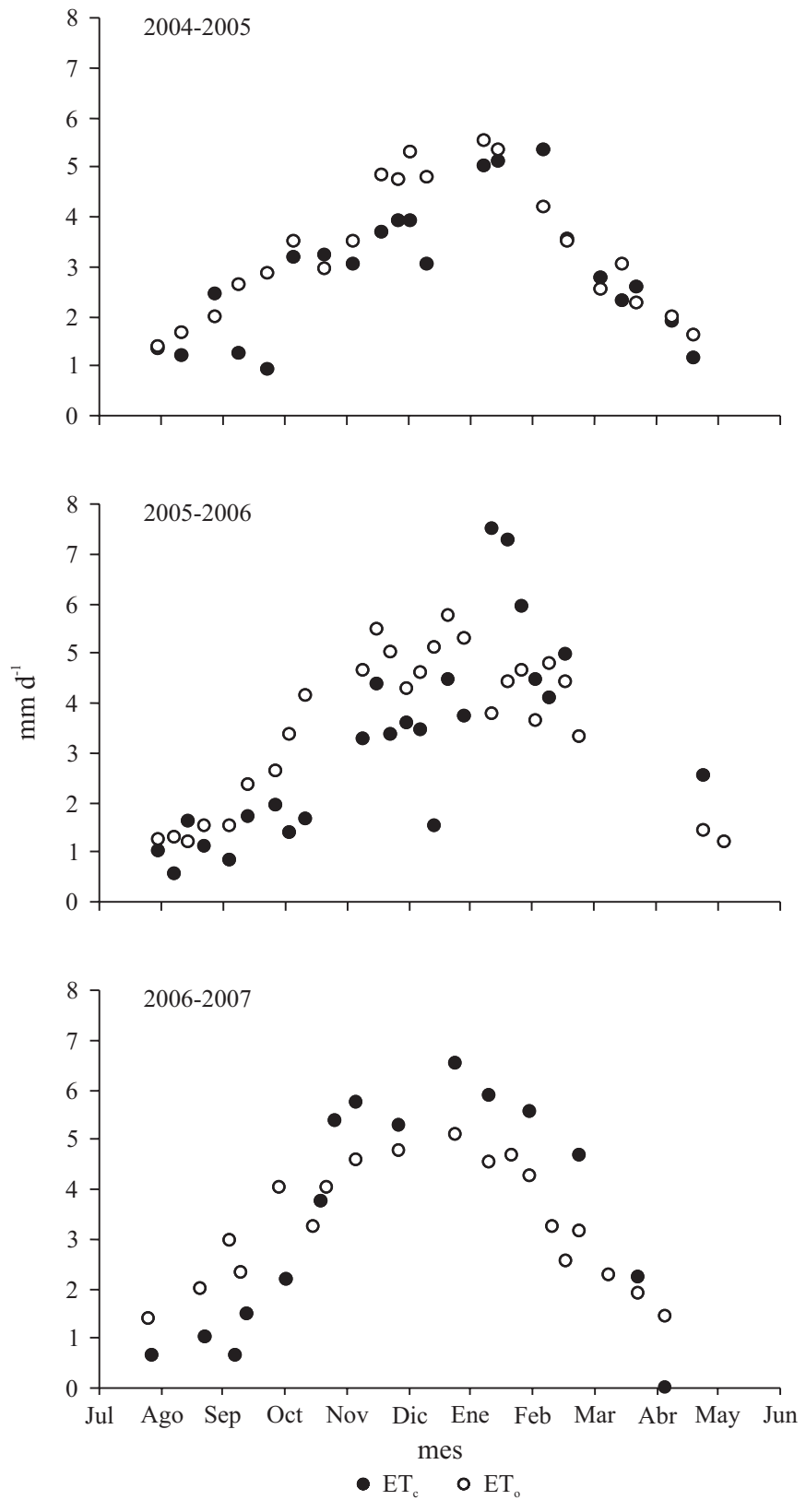

Figura 1. Evapotranspiración del duraznero (ETc) y Evapotranspiración de referencia (ETo) en las tres temporadas estudiadas

a alrededor de $6 \mathrm{~mm} \mathrm{~d}^{-1}$ (68 L), aunque en la segunda quincena de enero alcanzó valores extremos de más de $7 \mathrm{~mm} \mathrm{~d}^{-1}$, equivalentes a más de 80 L. Estos valores de consumo concuerdan con los reportados por Ayars et al. (2003) para árboles adultos de una variedad tardía de duraznero en California. Sin embargo, Paço et al. (2006) reportaron menores valores de consumo, 3,3 $\mathrm{mm} \mathrm{d}^{-1}$, con valores de ETo de 2,5-2,6 mm d d $^{-1}$ y IAF de 1,4, también más bajos que los correspondientes a este trabajo y que explicarían esta diferencia. El alto consumo de ese período posiblemente se debió a un incremento en la evaporación directa desde el suelo como consecuencia de las importantes lluvias ocurridas, $86 \mathrm{~mm}$ el día 14 de enero y luego lluvias semanales de entre $10 \mathrm{y}$ 15 mm (Figura 2) y a que el árbol aún no había alcanzado el máximo IAF de la temporada (Figura 3).
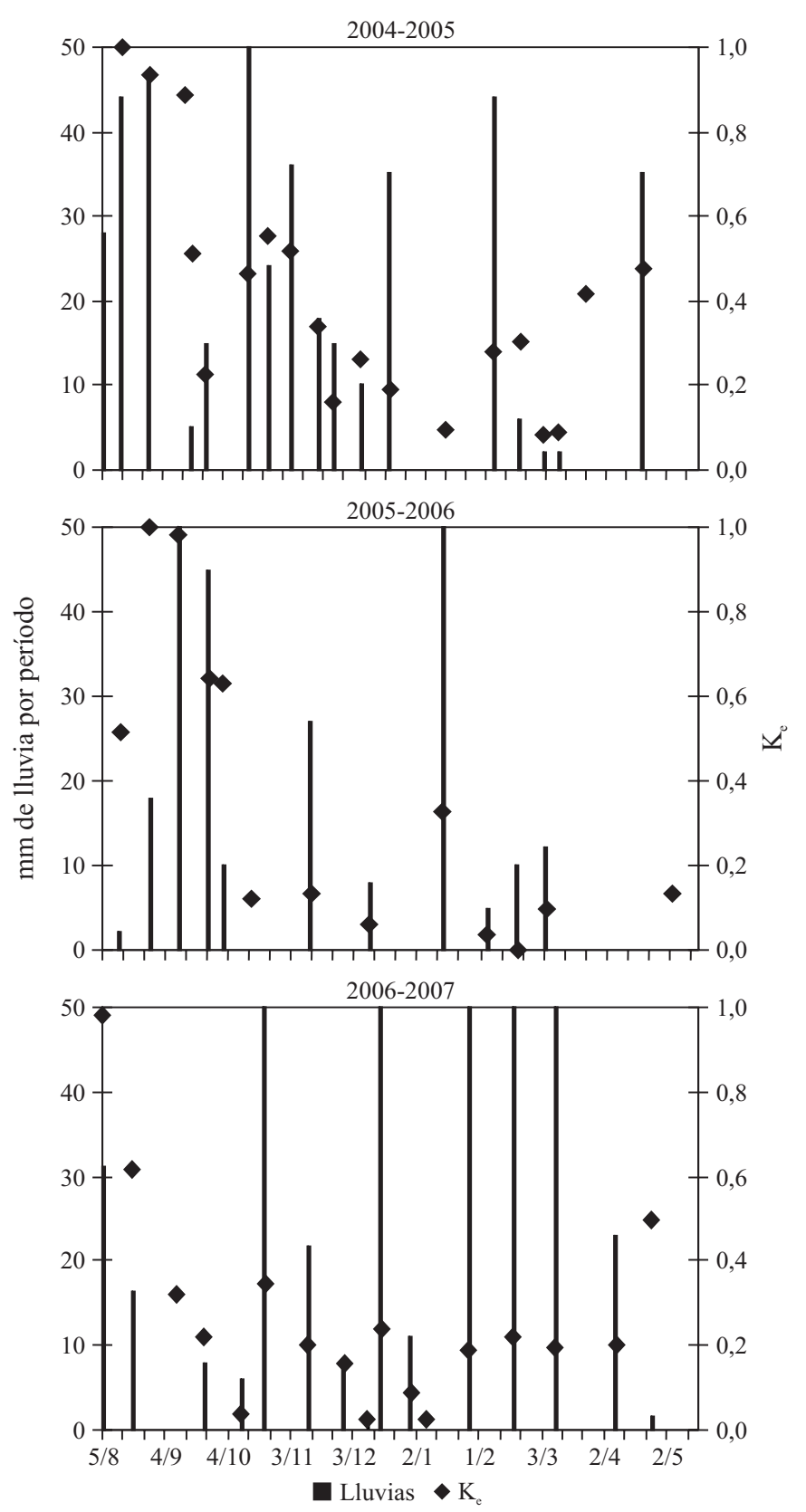

Figura 2. Lluvia acumulada y coeficiente de evaporación (Ke) ajustado por período en las tres temporadas estudiadas

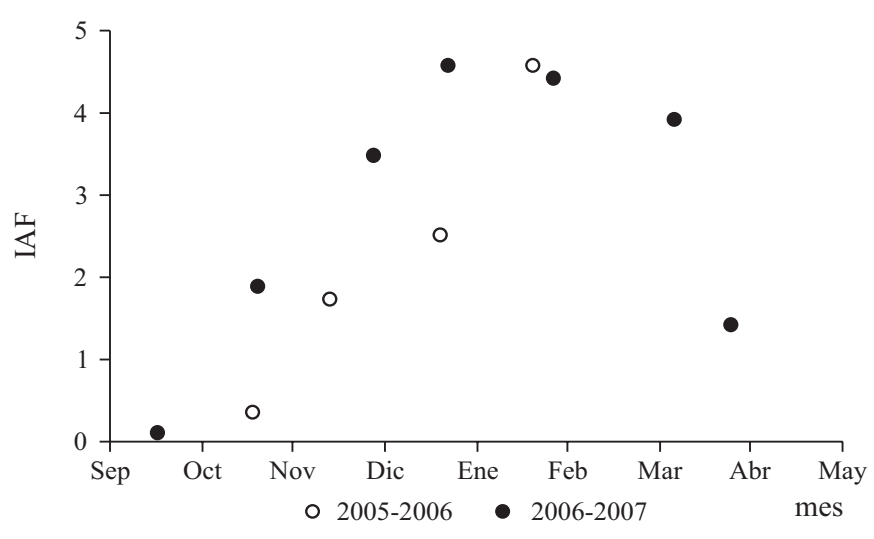

Figura 3. Evolución del Índice de Área Foliar (IAF), referido a todo el marco de plantación (4,5 x 2,5 m), en las temporadas 2005-2006 y 2006-2007 
En la tercera temporada (2006-2007), en que la planta ya alcanzó su tamaño adulto, el consumo máximo se mantuvo alrededor de los $6 \mathrm{~mm} \mathrm{~d}^{-1}$, aunque este valor se alcanzó más temprano que en la temporada anterior (segunda quincena de noviembre).

El valor de $68 \mathrm{~L} \mathrm{~d}^{-1}$ debería ser el parámetro de diseño de los equipos de riego para este cultivo en esta región.

Si se observa la evolución del área foliar (Figura 3) se ve que en la temporada 2006-2007 a fines de noviembre el IAF era de aproximadamente 3 , mientras que en la temporada anterior ese valor de IAF recién se alcanzó a mediados de enero.

Sin embargo, en ambas temporadas, se alcanza el mismo IAF máximo, de aproximadamente 4,6.

\section{Coeficientes de cultivo}

En la temporada 2004-2005, cuando la planta tenía sólo un año de edad, los coeficientes de cultivo $\left(\mathrm{K}_{\mathrm{c}}\right)$ tuvieron un valor aproximadamente constante a lo largo de toda la temporada y no reflejaron las fases de desarrollo del cultivo. En este primer año la mayor parte de la superficie del suelo del lisímetro estuvo expuesta a la radiación solar, por lo que al humedecerse esa superficie ante cada lluvia, los valores de coeficiente de evaporación $\left(\mathrm{K}_{\mathrm{e}}\right)$ fueron importantes (Figura 2), resultando en valores altos de $K_{c}$, aún en los inicios de la temporada de crecimiento, cuando la planta tenía muy poco desarrollo foliar (Figura 4).

En dicha Figura se observa que en la etapa inicial del desarrollo vegetativo del cultivo existen valores de Ke mayores que los valores de $\mathrm{K}_{\mathrm{c}}$. Esto, que es un error del punto de vista conceptual, ya que $\mathrm{K}_{\mathrm{c}}=\mathrm{K}_{\mathrm{e}}+\mathrm{K}_{\mathrm{cb}}$, se debe al método usado para determinar los coeficientes: el $\mathrm{K}_{\mathrm{c}}$ fue medido directamente en el lisímetro y el $\mathrm{K}_{\mathrm{c}}$ fue estimado mediante el cálculo teórico ya detallado.

A medida que el follaje de la planta se fue desarrollando, los valores de $\mathrm{K}_{\mathrm{e}}$ fueron decreciendo, para volver a aumentar hacia el final del ciclo del cultivo, coincidiendo con la senescencia del follaje (Figura 2).

En la segunda temporada de evaluación, se repitieron los altos valores de $\mathrm{K}_{\mathrm{e}}$ al inicio del ciclo del cultivo, que luego se mantuvieron bajos en el resto del ciclo (Figura 2). En la segunda quincena de enero se observaron valores extremadamente altos de $K_{c}(1,55)$, que coinciden con los valores extremos de consumo mencionados en el apartado anterior.

En la tercera temporada, se mantuvo un valor de $\mathrm{K}_{\mathrm{c}}$ alrededor de 1,4 desde noviembre hasta marzo.

Los $K_{c b}$ ajustados por el modelo tri-segmentado se presentan en la Figura 5.

Los máximos valores fueron 0,91; 1,04 y 1,20 para las temporadas 2004-2005 a 2006-2007, respectivamente. Esos valores se alcanzaron el día 30 de diciembre; 26 de enero y 24 de noviembre.

El aumento del valor del $\mathrm{K}_{\mathrm{cb}}$ de la primera a la segunda temporada se debió al incremento del tamaño de la planta. Sin embargo, a pesar que en la segunda y la tercera temporada el índice de área foliar (IAF) máximo alcanzado fue similar (Figura 3), el $\mathrm{K}_{\mathrm{cb}}$ fue mayor en la temporada 2006-2007. Los coeficientes de cultivo son afectados por las
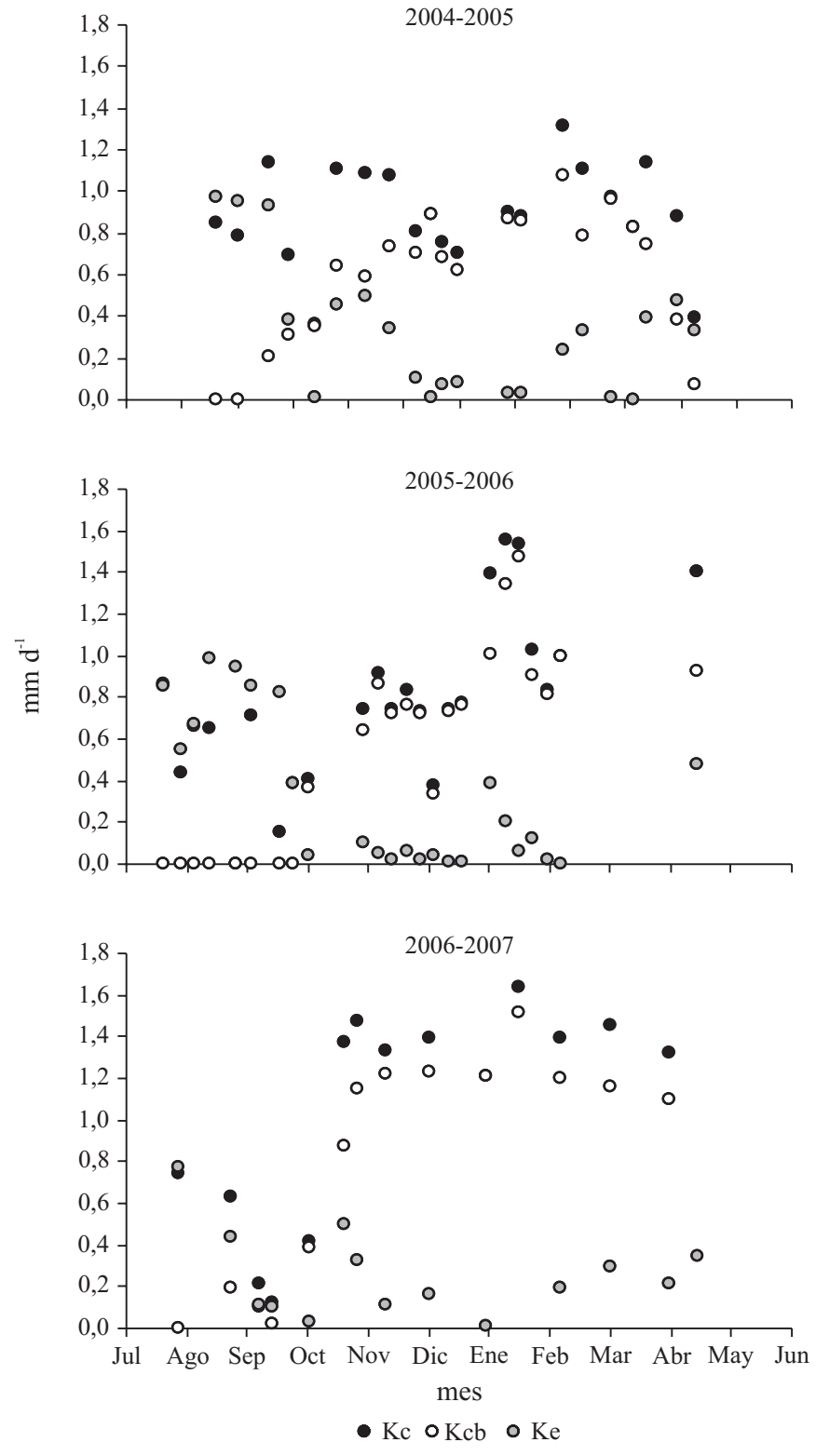

Figura 4. Coeficiente del cultivo $\left(K_{c}\right)$, Coeficiente de base $\left(K_{c b}\right)$ y Coeficiente de evaporación $\left(\mathrm{K}_{\mathrm{e}}\right)$ del duraznero, en las tres temporadas estudiadas

condiciones climáticas, aumentando su valor en la medida en que es mayor la velocidad del viento y menor la humedad relativa mínima (Allen et al., 1998). En este sentido, Johnson et al. (2004) encontraron que una gran parte de la variabilidad del $\mathrm{K}_{\mathrm{cb}}$ de duraznero era explicada por la variación del déficit de presión de vapor al mediodía. En la última temporada, se registraron valores más extremos tanto en la humedad relativa como en la velocidad del viento (datos no presentados), que explicarían este mayor $\mathrm{K}_{\mathrm{cb}}$.

En la temporada 2005-2006 el K cb máximo se alcanzó, de acuerdo al modelo, el día 26 de enero, mientras que en la temporada siguiente se alcanzó el 24 de noviembre. Este adelanto concuerda en ésta última temporada que al 24 de noviembre ya se había alcanzado un IAF de aproximadamente 3 (Figura 3), mientras que en la anterior ese índice se alcanzó recién a mediados de enero.

Estos resultados sugieren que habría un valor umbral del 

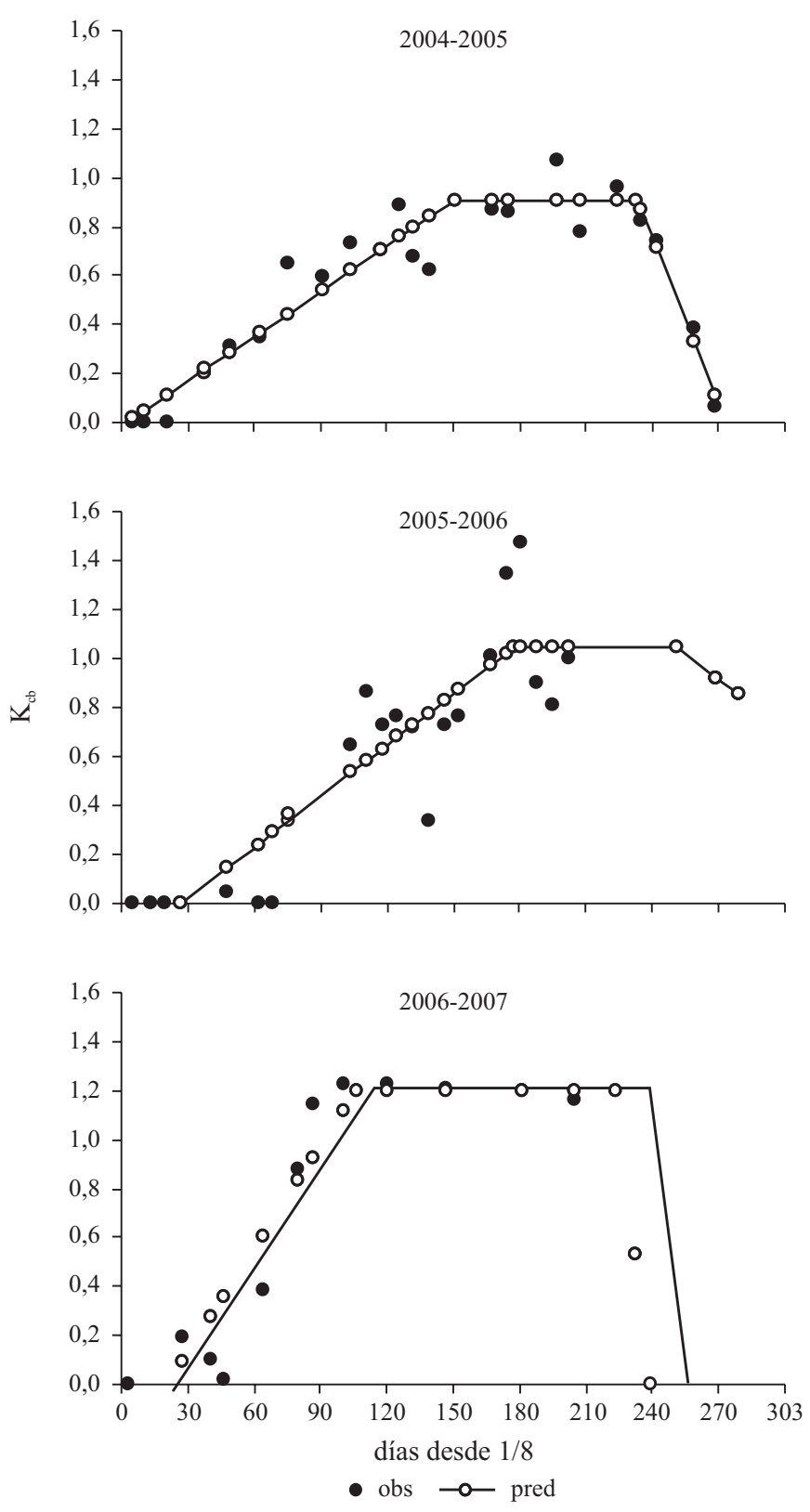

Figura 5. Coeficientes de base $\left(\mathrm{K}_{\mathrm{cb}}\right)$ observados y curva de ajuste usando el modelo tri-segmentado lineal - plateau - lineal, en las tres temporadas estudiadas

IAF, por encima del cual no seguiría aumentando el $\mathrm{K}_{\mathrm{cb}}$. Este valor sería aproximadamente 3 , pero para ajustar con más precisión la relación $\mathrm{K}_{\mathrm{cb}} / \mathrm{IAF}$, se deberá tener más información experimental.

\section{Ajustes metodológicos}

A los efectos de facilitar la realización de trabajos similares en el futuro, también se presentan como resultados algunos ajustes metodológicos surgidos de la práctica de estos tres años.

a. En los trabajos publicados por la Universidad de Pisa en los cuales se utiliza el mismo lisímetro que en esta investigación, se considera que el volumen de suelo siempre está a capacidad de campo (CC) o muy próxima a este contenido de agua, al comienzo de cada ciclo diario. Esta con- sideración resulta válida para los trabajos realizados, en su mayoría con cultivos hortícolas con profundidades de macetón de 0,40-0,50 m (Magnani et al., 1990; Bertolacci \& Megale, 1991). Para esta investigación, por tratarse de árboles frutales, se utilizó un macetón mayor, con una profundidad total de 0,80 m. En el primer año de desarrollo del árbol, se mantuvo el supuesto anterior. Sin embargo, en años sucesivos, con el desarrollo de la planta se constató que existían variaciones en el contenido de humedad a las diferentes profundidades del suelo. Debido a esto se debió incorporar el término de variación de la humedad en el balance para el cálculo de la $\mathrm{ET}_{\mathrm{c}}$. A estos efectos se recurrió a las lecturas de los tensiómetros ubicados a 15, 30 y $60 \mathrm{~cm}$ de profundidad y mediante la curva de retención se pudo contabilizar la $\Delta \mathrm{Hs}$.

b. En la última temporada, con la planta totalmente desarrollada, en los períodos de alta demanda atmosférica y con escaso o nulo aporte de las lluvias, el aporte de agua del lisímetro, por ascenso capilar de la napa, resultó insuficiente para satisfacer el consumo de la planta. Esto se evidenció en las mediciones de potencial hídrico y de conductancia estomática. Para solucionarlo, se aplicaron volúmenes medidos de agua desde la superficie del lisímetro.

c. La ecuación (2) para estimar la $\mathrm{ET}_{\mathrm{c}}$ se puede calcular diariamente. Sin embargo, los resultados así obtenidos resultaron algo erráticos, debido fundamentalmente a los procesos de redistribución de agua en el perfil, que se producen con retardo respecto a los aportes, principalmente lluvias. Por el contrario, al tomar períodos de cinco días o más, los resultados obtenidos fueron coherentes.

\section{CONCLUSIONES}

1. El consumo de una planta de duraznero en la zona sur del Uruguay es de $5 \mathrm{~mm} \mathrm{~d}^{-1}$ en su primer año y de $6 \mathrm{~mm} \mathrm{~d}^{-1}$ en el segundo y tercero, equivalentes a 56 y $68 \mathrm{~L} \mathrm{~d}^{-1}$, respectivamente.

2. Los valores de $K_{\mathrm{cb}}$ máximo obtenidos en las tres temporadas fueron superiores a los propuestos por FAO.

3. El máximo valor de $\mathrm{K}_{\mathrm{cb}}$ se alcanza cuando el Índice de Área Foliar llega a un valor de 3, y luego se mantiene constante aunque el IAF continúa aumentando.

4. En lisímetros de este tipo, y con este tamaño del macetón, se debe incluir el término $\Delta$ Hs en la ecuación de balance hídrico.

5. En condiciones de alta demanda atmosférica y la planta de tamaño adulto, se hace necesario regar para complementar el aporte por ascenso capilar de la capa.

6. Este método permite el ajuste del $\mathrm{K}_{\mathrm{cb}}$ para intervalos de tiempo mayores a cinco días.

\section{AgRADECIMIENTOS}

Al Ing. Agr. Jorge Franco, del Departamento de Estadística y Cómputos de la Facultad de Agronomía, por su apoyo en el ajuste del modelo tri-segmentado. 
Al Ing. Pier Gino Megale y al Laboratorio Nazionale dell Irrigazione. P. Celestre. de la Universidad de Pisa, por la donación del microlisímetro, y su generoso y constante apoyo.

\section{LITERATURA CITADA}

Aboukhaled, A. A.; Alfaro, A.; Smith, M. Los lisímetros. Roma: FAO, 1986. 68p. Paper 39.

Allen, R. G.; Clemmens, A. J.; Burt, C. M.; Solomon, K.; O’Halloran, T. Prediction accuracy for projectwide evapotranspiration using crop coefficients and reference evapotranspiration. Journal of Irrigation and Drainage Engineering, v.131, p.24-36, 2005a.

Allen, R. G.; Pereira, L. S.; Raes, D.; Smith, M. Crop evapotranspiration - Guidelines for computing crop water requirements. Rome: FAO, 1998. 300p. Paper 56.

Allen, R. G.; Pereira, L. S.; Smith, M.; Raes, D.; Wright, J. L. FAO-56 Dual crop coefficient method for estimating evaporation from soil and application extensions. Journal of Irrigation and Drainage Engineering, v.131, p.2-13, 2005b.

Ayars, J. E.; Johnson, R. S.; Phene C. J.; Trout T.; Clark D. A.; Mead, R. Water use by drip-irrigated late-season peaches. Irrigation Science, v.22, p.187-194, 2003.

Bertolacci, M.; Megale, P. G. Automazione degli impianti irrigui con microlisimetri. Colture Protette, v.6, p.CCV-CCVIII, 1991.

Boland, A. M.; Mitchel, P. D.; Jerie, P. H.; Goodwin, I. The effect of regulated deficit irrigation on tree water use and growth of peach. Journal of Horticultural Science, v.68, n.2, p.261-274, 1993.

García-Petillo, M.; Castel, J. R. Water balance and crop coefficient $\left(\mathrm{K}_{\mathrm{c}}\right)$ estimation of a citrus orchard in Uruguay. Spanish Journal of Agricultural Research, v.5, n.2, p.232-243, 2007.
García-Petillo, M.; Puppo, L.; Romero, G.; Baccino, G. Respuesta al riego de duraznero, manzano y peral en montes comerciales. Revista Agrociencia, v.VII, n.2, p.49-61, 2003.

Godinho, P.; Sequeira, B.; Paredes, P.; Pereira L. S. Simulação das necessidades de água das culturas pela metodologia dos coeficientes culturais duais. Modelo SIMDualKc. In: Taller Modernización de riegos y usos de tecnologías de información. Redes de Riegos de CYTED y PROCISUR, 2007, La Paz. Anais... 2007. CD Rom.

Goodwin I.; Whitfield D. M.; Connor D. J. Effects of tree size on water use of peach (Prunus persica L. Batsch). Irrigation Science, v.24, p.59-68, 2006.

Hsiao, T. C. Measurements of plant water status. In: Stewart, B. A.; Nielsen, D. R. (ed.) Irrigation of agricultural crops, Madison: ASA-CSSA-SSSA, n.30, 1990. Agronomy Monograph, p.244-279,

Johnson, R. S.; Ayars, J.; Hsiao, T. Improving a model for predicting peach tree evapotranspiration. Acta Horticulturae, n.664, p.341-346, 2004.

Magnani, G.; Bertolacci, M.; Oggiano, N.; Megale, P. G.; Cecarelli, E. Ottimizzazione della tecnica irriga in tunnel coperti con diversi materiali plastici rigidi (PMMA, PVC e PRFV). Colture Protette, v.7, p.25-32, 1990.

Paço, T. A.; Ferreira, M.; Conceiçao, N. Peach orchard evapotranspiration in a sandy soil: comparison between eddy covariance measurements and estimates by the FAO 56 approach. Agricultural Water Management, v.85, p.305-313, 2006.

Pereira, L. S. Necesidades de agua e métodos de rega. Mem Martins. Lisboa: Publicações Europa-América Lda., 2004. 312p.

Wright, J. L. New evapotranspiration crop coefficients. Journal of Irrigation and Drainage, ASCE, v.108, p.57-54, 1982. 